\title{
First clinical experiences with a novel endoscopic over-the-scope clip system $\square$
}

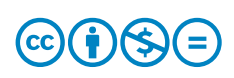

\author{
Authors \\ Marco Dinelli ${ }^{1}$, Barbara Omazzi², Paolo Andreozzi², Nicola Zucchini ${ }^{3}$, \\ Alessandro Redaelli ${ }^{1}$, Gianpiero Manes ${ }^{2}$ \\ Institutions \\ 1 Gastroenterology and Endoscopy Unit, San Gerardo Hospital, \\ Monza, Italy \\ 2 Department of Gastroenterology, ASST Rhodense, Rho and \\ Garbagnate Milanese Hospital, Milan, Italy \\ 3 Department of Pathology, San Gerardo Hospital, Monza, Italy
}

submitted 29.8.2016

accepted after revision $\quad 30.12 .2016$

Bibliography

DOI http://dx.doi.org/10.1055/s-0043-101692 |

Endoscopy International Open 2017; 05: E151-E156

(c) Georg Thieme Verlag KG Stuttgart · New York

ISSN 2364-3722

\author{
Corresponding author \\ Marco Dinelli, Gastroenterology and Endoscopy Unit, San Gerardo \\ Hospital, Via Giambattista Pergolesi, 33, 20900 Monza MB, Italy \\ Fax: +39-039-2332131 \\ m.dinelli@asst-monza.it
}

\begin{abstract}
We describe our experience with a new over-the-scope clip (OTSC) system (Padlock Clip ${ }^{\mathrm{TM}}$ ) in the treatment of 14 patients. Eight of the 14 patients were treated for closure of gastrointestinal fistulas $(n=$ $4)$, iatrogenic gastrointestinal perforations $(n=2)$, or hemostasis of post-polypectomy bleeding $(n=2)$. The site of clipping was the lower gastrointestinal tract in five patients and the upper gastrointestinal tract in three patients. The clip was successfully delivered in seven out of the eight patients and clinical success was achieved in all patients. Endoscopic full thickness resection (EFTR) was performed to treat six patients: four with recurrent adenoma $(n=4)$, one with ulcerated nodules at ileorectal anastomosis, and one with a neuroendocrine tumor of the rectum. A complete intestinal wall resection was achieved in three of the six patients $(50 \%)$ and an $\mathrm{R} 0$ resection in five of the six patients $(83.3 \%)$. No complications related to the procedure and no recurrence at endoscopic follow-up were observed in any patient. The novel Padlock Clip seems to be an effective and safe tool to treat gastrointestinal fistulas, perforations or post-polypectomy bleeding, and to perform EFTR.
\end{abstract}

\section{Introduction}

The increased invasiveness of endoscopic procedures has resulted in a higher number of complications, such as bleeding or perforations. Although surgery still represents the mainstay in the management of these complications, new endoscopic devices have been developed to allow endoscopic treatment of fistulas, perforations, and post-polypectomy bleeding. The Ovesco over-the-scope clip (OTSC) system (Ovesco Endoscopy AG, Tübingen, Germany) has shown promising results in the treatment of gastrointestinal fistulas [1], iatrogenic perforations [2], and bleeding [3,4]. In addition, the Ovesco OTSC has been proposed for endoscopic full thickness resection (EFTR) of epithelial and submucosal tumors [5, 6].

More recently, the Padlock Clip ${ }^{\mathrm{TM}}$ (Aponos Medical, Kingston, NH, United States), a new OTSC device, has been introduced into clinical practice. This device is conceptually similar to the Ovesco system, but has some features which make it very attractive. Few studies have reported on the efficacy of this device in clinical practice [7 -9]. Considering the emerging role of these devices in clinical practice, we decided to describe our experiences with this novel OTSC system in the treatment of gastrointestinal wall defects and EFTR.

\section{Case series}

We describe the results of the treatment of 14 consecutive patients (eight men, six women, 63-86 years) with the Padlock Clip over an 18-month period in two endoscopy units. Indications for clipping were: (i) gastrointestinal fistulas or iatrogenic perforations, (ii) gastrointestinal bleeding but non-responder to thermal and/or mechanical treatments, and (iii) EFTR of colonic lesions with non-lifting sign. All patients provided informed consent to undergo endoscopy, and re-endoscopy was performed according to clinical follow-up.

The Padlock Clip is an $11 \mathrm{~mm}$ hexagonal nitinol ring with six inner prongs. The clip is released by its Lock-It' ${ }^{\mathrm{TM}}$ delivery system that consists of an applicator cap, similar to the variceal bandligation cap ( $\mathbf{F i g . 1}$ ). The applicator cap is available in two models: (1) Standard for endoscopes with a diameter of distal tip between 9.5 and $11 \mathrm{~mm}$, and (2) Pro-Select ${ }^{\mathrm{TM}}$ for endoscopes with a diameter of distal tip between 11.5 and $14 \mathrm{~mm}$. In the Standard model, the depth of the tissue chamber is fixed $(10 \mathrm{~mm})$, whereas in the Pro-Select ${ }^{\mathrm{TM}}$ model, the depth of the tissue chamber depends on the diameter of the distal tip of the scope, ranging from $8 \mathrm{~mm}$ for $12 \mathrm{~mm}$ scopes to $20 \mathrm{~mm}$ for $14 \mathrm{~mm}$ scopes. In our series, we used the Standard model for 


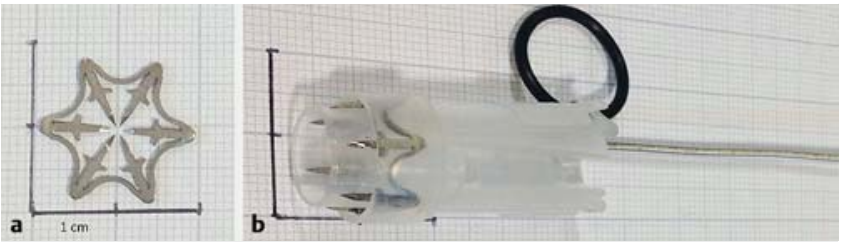

- Fig. 1 The Padlock Clip (a) and the Lock-It ${ }^{\mathrm{TM}}$ delivery system (b).

gastroscopes and the Pro-Select ${ }^{\mathrm{TM}}$ model for standard colonoscopes.

The clip is preassembled in an opened position on the delivery system and it is released by a trigger cable running parallel to the scope that is connected to a handle ( $\triangleright$ Video 1 ). After suction of tissue into the cap to obtain adhesion to the instrument tip, the clip is released by pressing the handle which pushes the wire within the cable. When deployed, the shapememory effect and the high elasticity of nitinol cause the closure of the clip. Its six inner prongs penetrate and pull the tissue inward resulting in a pseudo-polyp of closed tissue ( $>$ Fig. 2 and - Video 2). When performing EFTR, the entrapped tissue is resected with an oval snare above the closed clip by applying cut- ting current ( $\triangleright$ Fig. 3 and $\triangleright$ Video 3 ). Patients undergoing EFTR have been hospitalized for one night and then discharged the day after if asymptomatic. Technical success was defined as closure of the clip and the successful resection of the trapped tissue; specimens were classified as: "deep muscle resection" (DMR) when a deep muscle margin was present at the vertical margin; "full thickness resection" (FTR) if complete intestinal wall was included; $R 1$ resection if neoplastic tissue was still evident at the lateral margins, and $\mathrm{R} 0$ resection if no neoplastic remnant was evident.

The characteristics of the eight patients with fistulas, perforations, or post-polypectomy bleeding are summarized in

- Table 1. Indications for clipping were closure of gastrointestinal fistulas $(n=4)$, management of iatrogenic gastrointestinal perforations ( $n=2)$, or hemostasis of post-polypectomy bleeding $(n=2)$. Three clips were applied in the upper gastrointestinal tract (stomach and duodenum) and five clips in the lower gastrointestinal tract (rectum and sigmoid colon). In one patient, the delivery system failed to release the clip into the duodenum and the wall defect was closed with two through-thescope (TTS) clips. Clinical success (healing of defect or resolution of bleeding) was achieved in all patients. In three patients, clinical follow-up required re-endoscopy and no clip retention
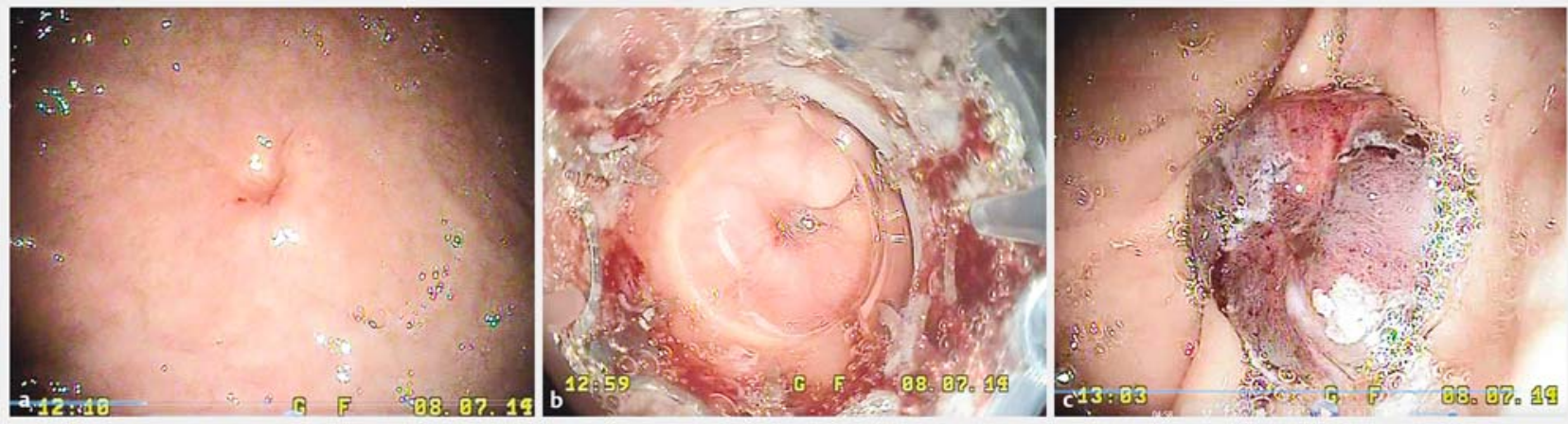

Fig. 2 Persistence of gastrocutaneous fistula after percutaneous endoscopic gastrostomy removal (a). The gastric wall is going to be aspirated within the cap (b). The deployed Padlock Clip entraps the gastric wall and closes the fistula (c).

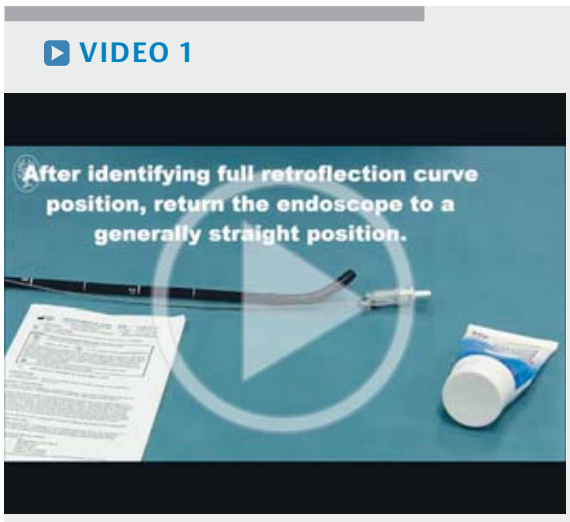

- Video 1: The Padlock Clip mounting.

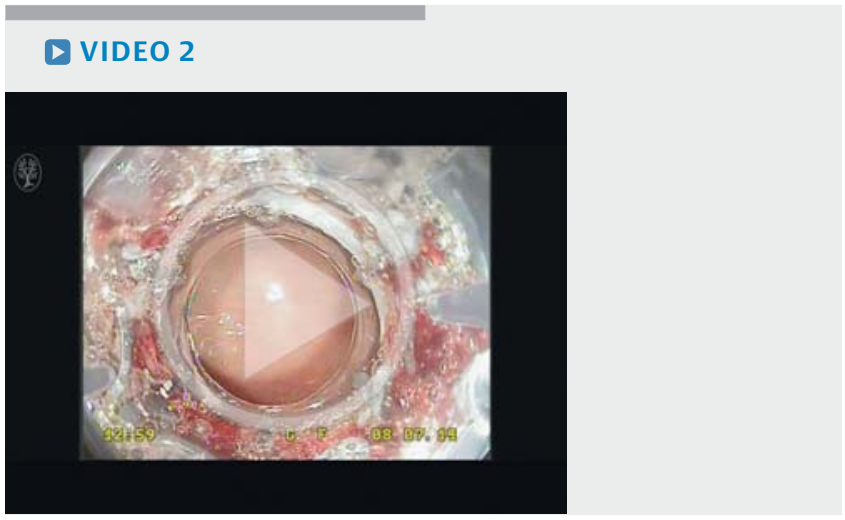

- Video 2: Closure of gastrocutaneous fistula with the Padlock Clip. 

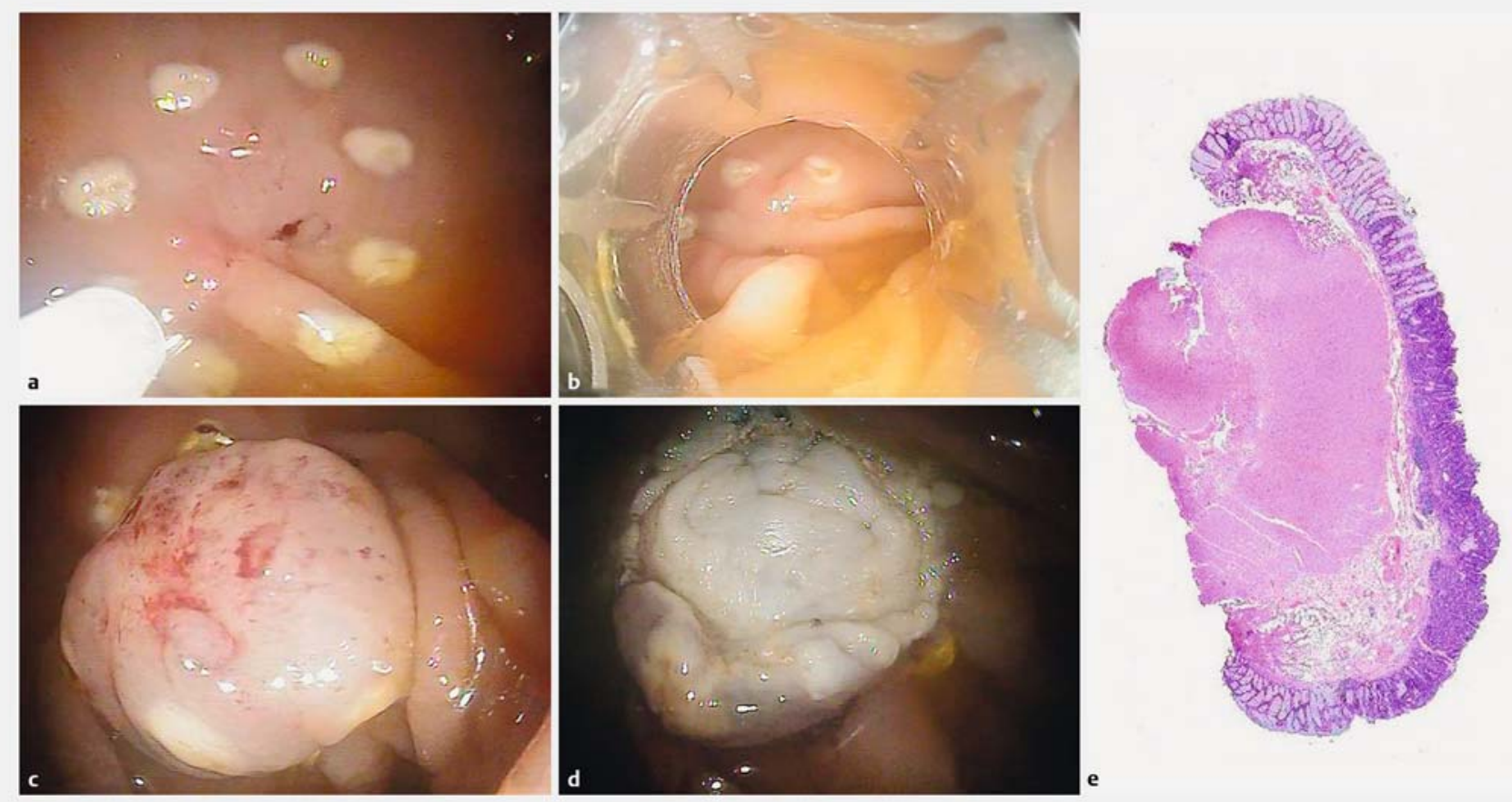

- Fig. 3 Rectal depressed lesion Ilc with non-lifting sign. After marking the borders (a), the lesion is aspirated into the cap (b). The pseudo-polyp resulting from entrapment of gastrointestinal wall into the clip (c) is resected with a snare (d). Histology demonstrates adenoma with high grade dysplasia with clear mucosal lateral margins (e). The muscular layer is well represented in the specimen.

was found. No related adverse events were observed in any of the eight patients.

In $>$ Table 2, we report the features of the six patients who underwent EFTR. In four of the six patients, the indication was a recurrent adenoma after endoscopic mucosal resection (EMR, one in the rectum, one in the sigmoid colon, and one in the ascending colon) or transanal endoscopic microsurgery (TEM, one patient); in one patient, two ulcerated nodules were removed using two clips at ileorectal anastomosis in a patient operated for colon cancer; and in the last case, a depressed scar in the rectum was removed; it had resulted from previous forceps resection of a small infiltrating neuro-endocrine tumor. Techni-

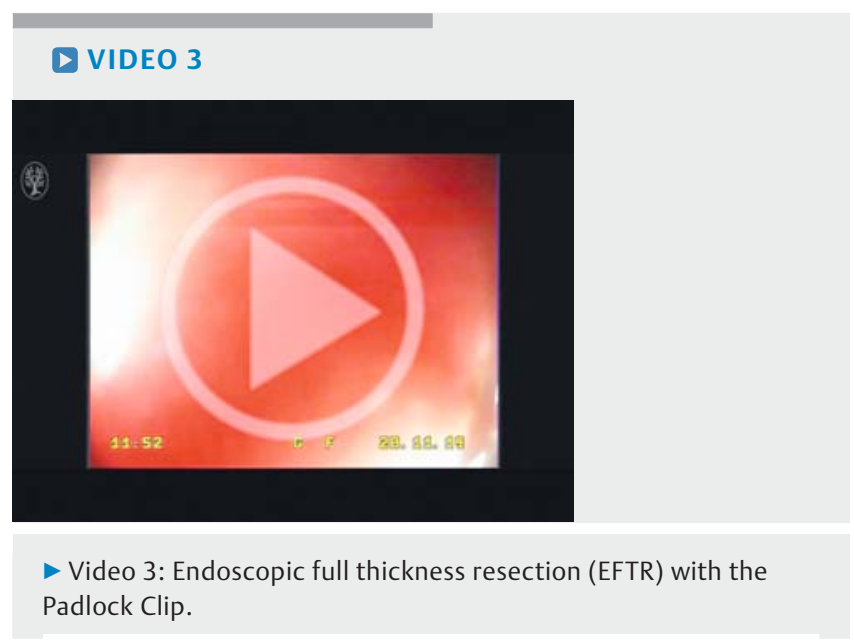

cal success was recorded in all six patients. In five patients, we obtained an R0 resection as defined by histology. In the case of anastomotic recurrence of adenocarcinoma, an R1 resection was obtained, but no recurrence was seen at 6 -month followup endoscopy. Histology demonstrated a DMR resection in three patients and a FTR in the remaining three. The mean diameter of the resected lesions was $12.5 \mathrm{~mm}$ (range $10-16 \mathrm{~mm}$ ). No complications were recorded. Follow-up ranged from 3 to 15 months and no recurrence was demonstrated either macroscopically or microscopically when the scars were biopsied. No clips were found at the first endoscopic control performed after 3 months.

\section{Discussion}

The novel Padlock Clip is conceptually similar to the Ovesco clip system, but Padlock's atypical design is likely to offer some advantages. In particular, the six inner prongs of the Padlock system, which are placed in a circumferential fashion, create a firm hold of the tissue which is easily lifted within the cap without the need for further devices such as forceps or graspers. Moreover, the system allows a thorough closure as demonstrated by an ex vivo study in explanted porcine stomachs where gas inflation revealed a mean leak pressure of $68 \mathrm{mmHg}$ [10].

In eight out of 14 patients, the Padlock was used to manage wall defects and post-polypectomy intraprocedural bleeding. Successful OTSC deployment was achieved in all but one patient, a patient with an iatrogenic perforation of the duodenum that was then successfully closed with two through-the-scope 


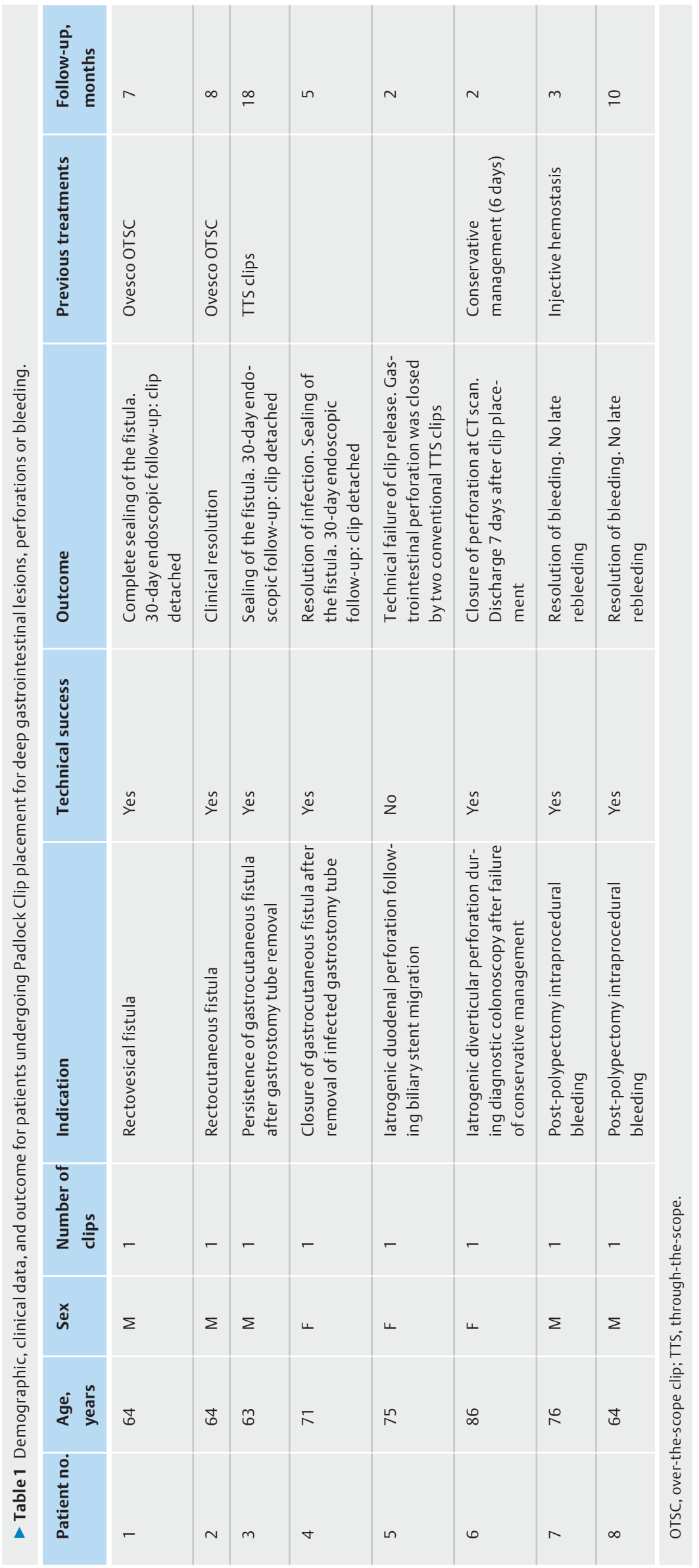




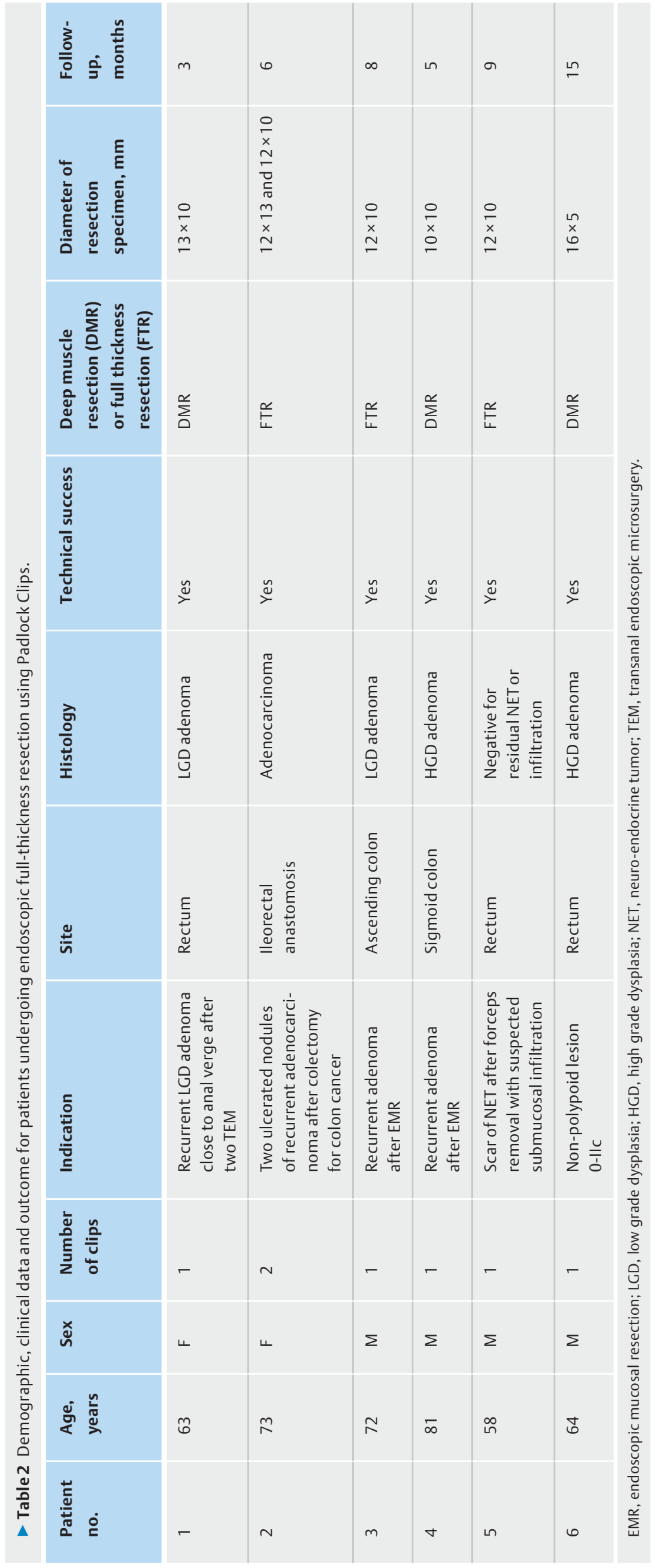

Dinelli Marco et al. First clinical experiences ... Endoscopy International Open 2017; 05: E151-E156

E155 
clips. Technical failure of clip release was probably due to the angulated position of the target site. In the other seven patients, the Padlock clipping resulted in long-term clinical success, with definitive closure of the gastrointestinal defects and resolution of intraprocedural bleeding. Interestingly, in our series, the Padlock clip was used successfully to treat two gastrointestinal fistulas after the application of an Ovesco clip. Although no comparison studies between the two systems are available, Padlock seems to be a reliable option in the management of gastrointestinal fistulas.

We describe for the first time the use of the Padlock system to perform EFTR in six patients, obtaining a FTR in $50 \%$ of patients attempted and at least a DMR in the remaining $50 \%$. We were able to achieve an $\mathrm{R} 0$ resection in five out of the six $(83.3 \%)$ patients without any complications. In the single case in which resection was considered incomplete, surgery was not indicated. Although we could not exclude the presence of residual tumoral cells buried within the closure of the clip, no local recurrence was revealed by endoscopy with random biopsies at 6-month follow-up.

Fahndrick and Sandmann [5] performed 17 EFTR (14 in the colon) without any complications using the standard Ovesco clip, and achieved a complete resection in $70 \%$ of patients, a FTR in $64 \%$ and an R0 resection in $100 \%$. Schmidt et al. [6] used the dedicated Ovesco device for EFTR to perform resection in 25 patients (to diagnose Hirschsprung's disease in one patient). In one patient, the progression of the scope was hindered by a diverticular stricture of the sigmoid colon; in 20 out of 24 patients, the EFTR was successful, with histologically proven FTR in 21 patients and $R 0$ resection in 18 patients. Two burning syndromes and one minor bleeding were reported. In all these patients, a grasping device was used to pull the tissue into the cap. In our application of the Padlock Clip, we did not use any grasping forceps (although it is theoretically possible). In addition, the diameter of the Padlock Clip and the length of the cap are smaller than in the dedicated Ovesco device for EFTR. These differences explain the smaller size of the specimens compared to the size of the specimens in the Schmidt study [6]. A combination of grasping and aspiration needs to be tested in a larger series and clinical trials to improve the outcomes of Padlock Clip-assisted EFTR.

The main limitations of our study were the small number of patients and the nature of the study (non-controlled design, variable indications). Despite these limitations, the new Pad- lock system seems to be an effective and safe tool, even when it is used to perform EFTR. However, further prospective clinical studies are needed to confirm its efficacy and safety, as well as to evaluate its correct use in clinical practice and explore new clinical indications.

\section{Competing interests}

None

References

[1] Manta R, Manno M, Bertani H et al. Endoscopic treatment of gastrointestinal fistulas using an over-the-scope clip (OTSC) device: case series from a tertiary referral center. Endoscopy 2011; 43: 545-548

[2] Donatelli G, Dumont JL, Vergeau BM et al. Colic and gastric over-thescope clip (Ovesco) for the treatment of a large duodenal perforation during endoscopic retrograde cholangiopancreatography. Ther Adv Gastroenterol 2014; 7: 282-284

[3] Manta R, Galloro G, Mangiavillano B et al. Over-the-scope clip (OTSC) represents an effective endoscopic treatment for acute GI bleeding after failure of conventional techniques. Surg Endosc 2013; 27: $3162-3164$

[4] Wedi E, Gonzalez S, Menke D et al. One hundred and one over-thescope-clip applications for severe gastrointestinal bleeding, leaks and fistulas. World J Gastroenterol 2016; 22: $1844-1853$

[5] Fahndrich M, Sandmann M. Endoscopic full-thickness resection for gastrointestinal lesions using the over-the-scope clip system: a case series. Endoscopy 2015; 47: $76-79$

[6] Schmidt A, Bauerfeind P, Gubler C et al. Endoscopic full-thickness resection in the colorectum with a novel over-the-scope device: first experience. Endoscopy 2015; 47: 719-725

[7] Armellini E, Crino SF, Orsello M et al. Novel endoscopic over-the-scope clip system. World J Gastroenterol 2015; 21: 13587-13592

[8] Diana M, Swanstrom LL, Halvax P et al. Esophageal covered stent fixation using an endoscopic over-the-scope clip. Mechanical proof of the concept and first clinical experience. Surg Endosc 2015; 29: $3367-3372$

[9] Brindley JH, Yip B, Vlachou E et al. Successful endoscopic closure of a gastrocutaneous fistula using a 'Padlock Clip'. Endoscopy 2016; 48: (Suppl. 01): E115-116

[10] Desilets DJ, Romanelli JR, Earle DB et al. Gastrotomy closure with the lock-it system and the Padlock-G clip: a survival study in a porcine model. J Laparoendosc Adv Surg Tech Part A 2010; 20: 671-676
NOTE

Ovesco Endoscopy AG has notified us that the "OTSC" designation used in the article is a registered trademark. 